\title{
Helicobacter pylori CagA upregulation of CIP2A is dependent on the Src and MEK/ERK pathways
}

Correspondence

Jihui Jia

jiajihui@sdu.edu.cn

Zhifang Liu

liuzhifang@sdu.edu.cn

Received 23 July 2009

Accepted 30 November 2009

\section{Dapeng Zhao, ${ }^{1} \dagger$ Zhifang Liu, ${ }^{2} \dagger$ Jian Ding, ${ }^{1}$ Wenjuan Li, ${ }^{1}$ Yundong Sun, ${ }^{1}$ Han Yu, ${ }^{1}$ Yabin Zhou, ${ }^{1}$ Jiping Zeng, ${ }^{2}$ Chunyan Chen $^{1}$ and Jihui Jia ${ }^{1}$}

\author{
${ }^{1}$ Department of Microbiology and Key Laboratory for Experimental Teratology of Chinese Ministry \\ of Education, School of Medicine, Shandong University, Jinan, PR China
}

${ }^{2}$ Department of Biochemistry, School of Medicine, Shandong University, Jinan, PR China

\begin{abstract}
Helicobacter pylori is classified as a class I carcinogenic factor and its persistent colonization in the stomach induces gastric cancer. Cancerous Inhibitor of PP2A (CIP2A) is a newly identified oncoprotein overexpressed in gastric cancer. Serving as a key oncoprotein, CIP2A also participates in regulation of senescence and proliferation of gastric cells. The combination of aberrant CIP2A expression inducing unlimited cell proliferation, and $H$. pylori infection eliciting aberrant expression of some key proteins, results in the onset of gastric tumorigenesis. However, the relationship between $H$. pylori infection and CIP2A expression still remains undefined. The aim of our study was to verify the effect of $H$. pylori infection on CIP2A expression levels and identify $H$. pylori signalling molecules and corresponding pathways influencing CIP2A expression. Following plasmid-mediated expression of CagA in human gastric cell lines, the cells were infected with $H$. pylori and CIP2A expression levels were examined by immunoblotting. Signal inhibitors were used to verify which signal pathways were involved. We also performed CIP2A depletion and $H$. pylori infection after depletion in AGS cells. $H$. pylori infection-induced CIP2A expression was dependent on cagA gene expression and CagA phosphorylation. Bacterial oncoprotein CagA upregulated CIP2A expression and this upregulation effect was dependent on Src and Ras/mitogen-activated protein kinase kinase/extracellular signal-regulated kinase pathways. H. pylori infection-induced Myc stabilization was partially attenuated by CIP2A depletion. The results of our study provide further information for understanding the mechanism of H. pylori carcinogenesis.
\end{abstract}

\section{INTRODUCTION}

Helicobacter pylori has been defined as a class I carcinogenic factor by the World Health Organization and its persistent colonization in the stomach leads to an increased risk of peptic ulcers and gastric adenocarcinoma (Houghton \& Wang, 2005; Peek \& Blaser, 2002). Some H. pylori strains contain a 35-40 kb cag pathogenicity island (PAI) encoded by 27-33 genes (Akopyants et al., 1998; Azuma et al., 2004). One constituent of the cag PAI is cagA, which encodes a 120-140 kDa CagA protein. CagA protein, which has tyrosine phosphorylation (EPIYA) motifs, is injected into gastric epithelial cells by the type IV secretory system (TFSS) (Odenbreit et al., 2000; Stein et al., 2000). cagA-

†These authors contributed equally to this work.

Abbreviations: CagA, cytotoxin-associated gene A; CIP2A, Cancerous Inhibitor of PP2A; MEK/ERK, Ras/mitogen-activated protein kinase kinase/extracellular signal-regulated kinase; PP2A, protein phosphatase 2A; PR, phosphorylation-resistant; siRNA, small interfering RNA; TFSS, type IV secretory system; WT, wild-type. positive $H$. pylori strains have a closer association with the progress of both peptic ulcers and gastric cancer than cagAnegative strains according to epidemiological research (Hatakeyama, 2003). Within gastric epithelial cells, tyrosine phosphorylation occurs at the C terminus of CagA by the Src family of tyrosine kinases (Kwok et al., 2007; Stein et al., 2002). CagA interacts with many signal molecules and elicits a series of cellular events. These events include activation of the Ras/mitogen-activated protein (MAP) kinase kinase (MEK)/ extracellular signal-regulated kinase (ERK) pathway, $\beta$ catenin, Src kinase, the NF- $\kappa$ B pathway and the p38 MAP kinase pathway. Some changes related to cell morphology, cell scattering, cell proliferation and intercellular tight junctions have also been identified (Hatakeyama, 2003, 2004; Nguyen et al., 2008; Backert \& Selbach, 2008; Backert \& Meyer, 2006; Handa et al., 2007). According to these observations, bacterial oncoprotein CagA participates in gastric tissue injury caused by cagA-positive $H$. pylori infection.

Unlimited cellular proliferation is a hallmark of tumours. Tumorigenesis is related to the disordered expression of 
some key factors which participate in the regulation of cell cycle progression, differentiation, apoptosis and senescence (Hanahan \& Weinberg, 2000). Recent studies have reported that CIP2A (Cancerous Inhibitor of PP2A) serves as an important oncoprotein (Junttila et al., 2007). Protein phosphatase 2A (PP2A) can facilitate the proteolytic degradation of oncoprotein Myc and prevent malignant cell growth (Yeh et al., 2004). CIP2A stabilizes Myc protein through inhibiting PP2A activity and promotes tumour formation in vivo (Junttila et al., 2007). Moreover, transformation of immortalized human cells is observed when CIP2A is overexpressed (Junttila et al., 2007). More importantly, overexpression of CIP2A has been observed in gastric cancer (Li et al., 2008). Using small interfering RNA (siRNA) to knock down CIP2A expression inhibits cell growth and clone formation, and promotes senescence in gastric cancer-derived AGS cell lines (Li et al., 2008). Taken together, CIP2A is a newly identified oncoprotein in gastric tissues, and may serve as a diagnostic marker and therapeutic target for gastric tumours (Li et al., 2008).

As mentioned above, $H$. pylori infection could affect the expression level of gastric carcinogenic factors, such as Myc (Zhu et al., 2008), $\beta$-catenin (Franco et al., 2005) and cyclin D1 (Chang et al., 2006). However, up to now, the relationship between $H$. pylori infection and CIP2A expression has never been studied. However, both bacterial oncoprotein CagA and cellular oncoprotein CIP2A expression have been associated with gastric carcinogenesis. To figure out the effect of CagA on CIP2A may help us to understand the carcinogenic mechanism of $H$. pylori infection and the functional role of CIP2A in human gastric tumorigenesis. In order to elucidate the relationship between these two factors, we designed $H$. pylori infection and CagA transfection in gastric cells and tried to investigate whether main virulence factor CagA of $H$. pylori could increase the expression of oncoprotein CIP2A in cell lines. Furthermore, we used signal inhibitors to examine which pathway was involved in the CagA-mediated regulation of CIP2A expression.

\section{METHODS}

H. pylori and bacterial culture. The H. pylori strains used in this study were cagA- and vacA-positive standard strains (NCTC 11637 and 26695). H. pylori NCTC 11637 has EPIYA-ABCCC type CagA, while H. pylori 22695 has EPIYA-ABC type CagA. Both strains were kindly provided by Dr Zhang Jianzhong (Chinese Disease Control and Prevention Center). Isogenic cag $A^{-}$mutants including cag $A^{-} 26695$ and $\operatorname{cag} A^{-} 11637$ were constructed within strains 11637 and 26695 by insertional mutagenesis using the piLL570 vector. All strains were grown in Brucella broth with $5 \%$ fetal bovine serum (FBS) under microaerobic conditions $\left(5 \% \mathrm{O}_{2}\right.$, $10 \% \mathrm{CO}_{2}, 85 \% \mathrm{~N}_{2}$ ) at $37{ }^{\circ} \mathrm{C}$, harvested by centrifugation, and added to gastric cells at a bacteria-to-cell ratio of $100: 1$.

Plasmid. Wild-type (WT) cagA/pcDNA3.1 (+) plasmid (WT-cagA) and a phosphorylation-resistant (PR) plasmid derivative (PR-cagA) were kindly provided by Zhu Yongliang (Zhejiang University, China). These two plasmids were characterized as described previously (Zhu et al., 2004, 2005). The pcDNA3.1(+) mammalian expression vector was purchased from Invitrogen.
Cell lines and culture conditions. The human gastric epithelial AGS cells and immortalized GES-1 cells were maintained in our laboratory. AGS gastric cancer cells were cultured in F12 medium (Gibco Life Technologies) with 10\% FBS (Gibco Life Technologies). GES-1 cells were cultured in RPMI 1640 medium (Gibco Life Technologies) with $10 \%$ newborn bovine serum (Gibco Life Technologies). All cells were cultured in $5 \% \mathrm{CO}_{2}$-air at $37{ }^{\circ} \mathrm{C}$.

Transfection of AGS and GES-1 cells. FuGENE HD Transfection Reagent (Roche Applied Science) was used to transfect plasmid WTcagA, PR-cagA or pcDNA3.1 blank vector into $5 \times 10^{5}$ AGS or GES-1 cells in six-well plates. All transfection procedures were performed according to the manufacturers and all experiments were repeated three times. After the transfection, cells were harvested and lysed using cell lysis buffer (Beyotime) supplemented with protease inhibitors (Complete Mini; Roche Applied Science) before freezing at $-80{ }^{\circ} \mathrm{C}$. In order to investigate the signal pathways in the epithelial cells, we used the following reagents: BAY11-7082 $(5 \mu \mathrm{M})$, PP1 $(20 \mu \mathrm{M})$, PD98059 $(50 \mu \mathrm{M})$ and SB203580 $(10 \mu \mathrm{M})$ (all from Invitrogen). We used one of these reagents to pre-incubate cells for $30 \mathrm{~min}$, and then transfected plasmids into cells. Protein collection methods were the same as without exposure to signal blocking reagents.

Western blot analysis. We used a BCA reagent kit (Merck) to measure protein concentrations and standardized samples in Western blot analysis according to the protein concentrations. Protein samples were separated by SDS-PAGE ( $8 \%$ SDS-acrylamide gels), and then proteins were transferred to a nitrocellulose membrane. After that, the membranes were incubated in the blocking buffer (TBS containing $0.1 \%$ Tween, $5 \%$ nonfat powdered milk) for $1.5 \mathrm{~h}$, and immunoblotted for $1.5 \mathrm{~h}$ with antibodies against Myc, $\beta$-actin, C-CagA (Santa Cruz Biotechnologies) or CIP2A (Novus Biologicals). Membranes were then washed three times with TBS-Tween solution and incubated with the corresponding antibodies conjugated to horseradish peroxidase for $50 \mathrm{~min}$. We then washed the membranes three times with TBS-Tween and used the Chemilucent ECL Detection System (Millipore) to detect the signals. Bio-Rad Quantity One 1-D Analysis software was used to analyse the Western blot results.

siRNA experiments. The siRNA targeting CIP2A and control siRNA were purchased from Invitrogen. The siRNA sequence for CIP2A was 5'-GACAACUGUCAAGUGUACCACUCUU-3'. Lipofectamine 2000 (Invitrogen) was used to transfect the gastric cells with siRNA.

Statistical analysis of data. The Statistical Package for the Social Sciences (SPSS) was used in the statistical analysis, and a Student's $t$ test was used to determine statistical significance. $P$-values $<0.05$ were considered statistically significant.

\section{RESULTS AND DISCUSSION}

\section{Effect of $\boldsymbol{H}$. pylori infection on CIP2A expression level in AGS cells}

H. pylori is a human gastric carcinogenic pathogen and CIP2A is a newly identified oncoprotein in gastric cancer (Li et al., 2008; Khanna et al., 2009). To elucidate whether $H$. pylori upregulated CIP2A expression, we designed $H$. pylori infection in human gastric epithelium AGS cells. AGS cells were incubated with $H$. pylori strain 26695 for $0.5,1,1.5$ and $2 \mathrm{~h}$, at a quantity of 100 bacteria per cell. Total cellular protein analysed by Western blotting indicated that the CIP2A expression level was elevated after infection for all time points compared to the expression in normal 
AGS cells, but that the effect was not remarkably timedependent (Fig. 1a). Furthermore, in order to elucidate which constituent of $H$. pylori is essential for promotion of CIP2A expression, we used $H$. pylori strains cagA ${ }^{-} 26695$, cag $A^{+} 26695, \operatorname{cag} A^{-} 11637$ and $\operatorname{cag} A^{+} 11637$ to infect AGS cells for 6 h. The CIP2A expression level was elevated only after infection with strains $c a g A^{+} 26695$ or $c a g A^{+} 11637$ compared to the expression in normal AGS cells (Fig. 1b). However, there was no significant increase in CIP2A expression level in AGS cells infected with strain $c a g A^{-} 26695$ and strain $c a g A^{-} 11637$ compared to the expression in normal AGS cells (Fig. 1b). Taken together, H. pylori infection increased CIP2A expression in AGS cells, and this effect was not dependent on infection time but dependent on expression of the bacterial $\operatorname{cag} A$ gene.

\section{CIP2A expression in AGS and GES-1 cells transfected with plasmid WT-cagA or PR-cagA}

As CIP2A expression was demonstrated to be associated with CagA expression of $H$. pylori, we next evaluated the relationship between CagA and CIP2A in the absence of $H$. pylori infection. AGS and GES-1 cells were transfected with plasmid WT-cagA or PR-cagA. The PR-cagA plasmid encodes a CagA protein with a mutation in the EPIYA motif, required for CagA tyrosine phosphorylation. Thus the PR-CagA mutant protein cannot be phosphorylated nor can it transmit the signal pathway (Zhu et al., 2004, 2005; Yokoyama et al., 2005). After $36 \mathrm{~h}$ transfection, the CIP2A expression level in AGS and GES-1 cells expressing WT-CagA was significantly greater than that in normal cells and cells transfected with blank vector (Fig. 2a). In contrast, there was no increase in CIP2A expression level in AGS and GES-1 cells transfected with vector expressing PR-CagA compared with cells alone or blank vector (Fig. 2b). Moreover, the Myc expression level was also elevated in cells expressing WTCagA (Fig. 2a). Antibody against C-CagA ensured that WT$\operatorname{cag} A$ and $\mathrm{PR}-\operatorname{cag} A$ plasmid transfections and CagA protein expression were accomplished as expected (Fig. 2a, b). Taken together, bacterial oncoprotein CagA can upregulate the expression of CIP2A and this effect is closely dependent on the tyrosine phosphorylation of CagA.

\section{Signal inhibitor effects on CIP2A expression in AGS cells transfected with WT-cagA plasmid}

After translocating into epithelial cells through the TFSS, H. pylori CagA protein is known to activate the MEK/ERK pathway, Src kinase, the NF- $\kappa \mathrm{B}$ pathway and the p38 MAP kinase pathway regardless of its tyrosine phosphorylation (a)
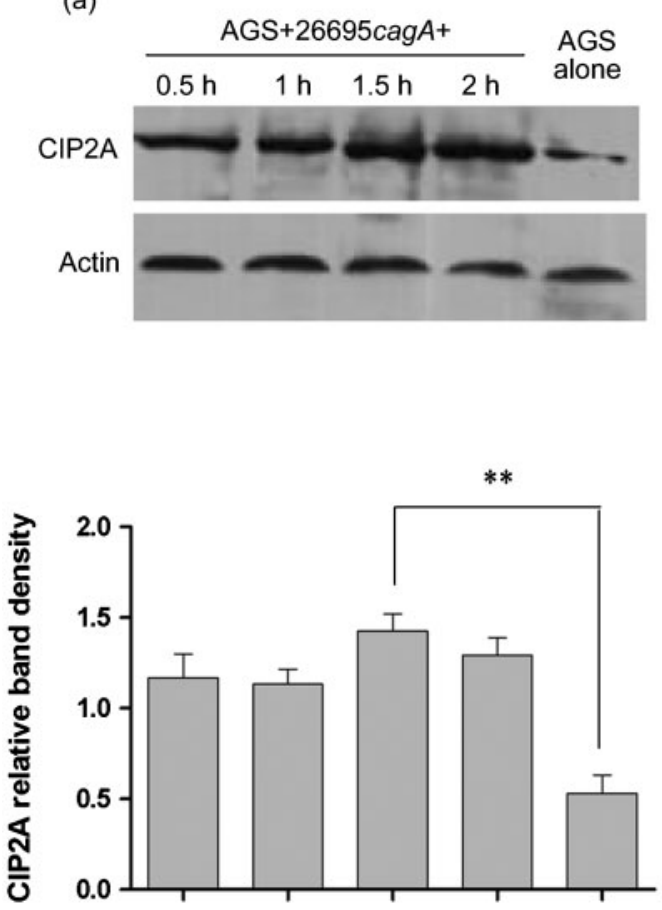

(b)
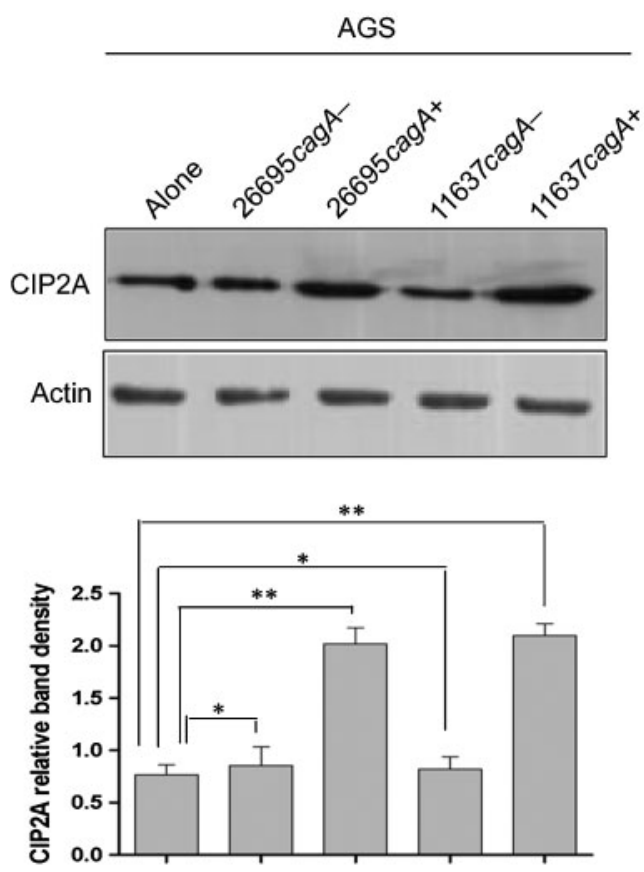

Fig. 1. Effect of $H$. pylori infection on CIP2A expression in AGS cells. (a) AGS gastric epithelial cells were incubated with medium alone, or with strain 26695 for 0.5 h, 1 h, 1.5 h or 2 h. Total cellular protein was extracted for Western blot analysis for the expression of CIP2A proteins. (b) AGS gastric epithelial cells were incubated with medium alone, or with strain $26695 \mathrm{cag}^{-}, 26695 \mathrm{cag} A^{+}, 11637 \mathrm{cag} A^{-}$or $11637 \mathrm{cag} A^{+}$for $6 \mathrm{~h}$. Total cellular protein was extracted for Western blot analysis for the expression of CIP2A. Representative results are shown. ${ }^{*} P>0.05,{ }^{\star *} P<0.01$. 
(a) GES-1
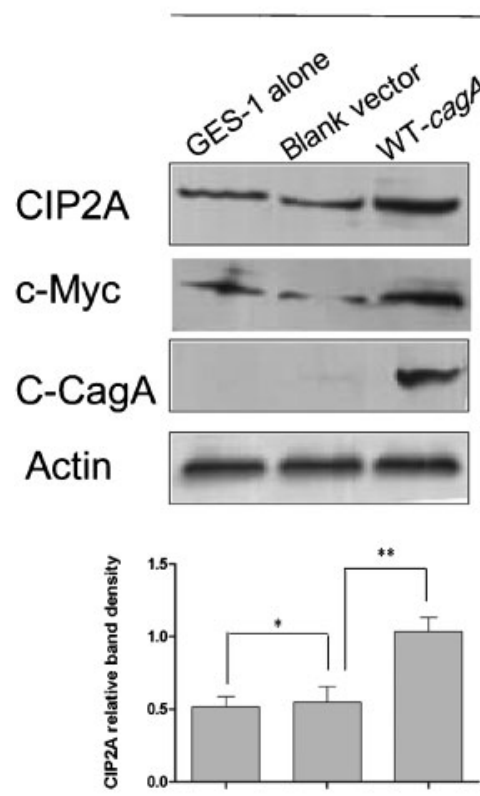

AGS
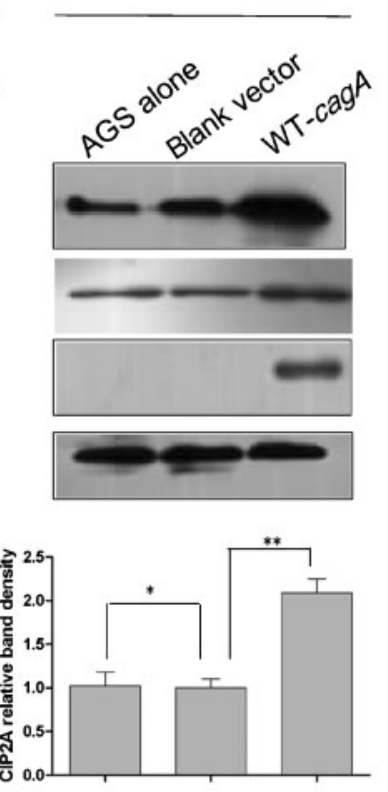

(b)

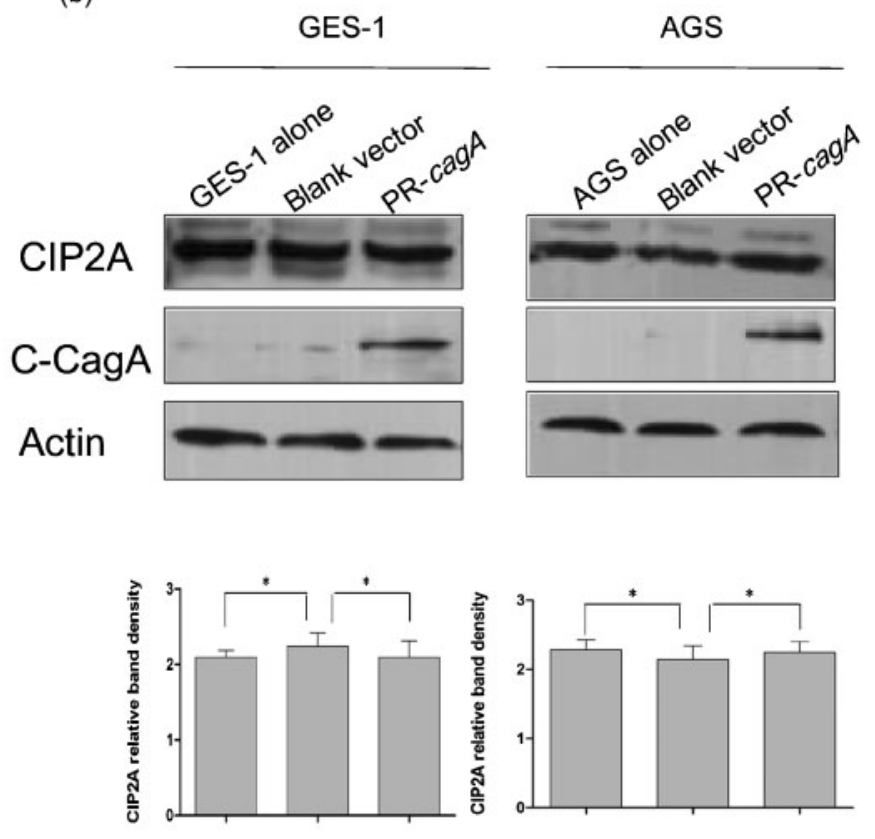

Fig. 2. Analysis of CagA transfection and its effects on CIP2A and Myc expression. (a) GES-1 and AGS gastric epithelial cells were transfected with blank vector pcDNA3.1 or WT-cagA plasmid for $36 \mathrm{~h}$, respectively. Total cellular protein was extracted for Western blot analysis for the protein expression of CIP2A, Myc and C-terminal CagA. (b) GES-1 and AGS gastric epithelial cells were transfected with blank vector pcDNA3.1 or PR-cagA plasmid for $36 \mathrm{~h}$, respectively. Total cellular protein was extracted for Western blot analysis for the expression of CIP2A and C-terminal CagA proteins. ${ }^{*} P>0.05$, ${ }^{\star \star} P<0.01$.

status. In order to elucidate which pathway participated in the upregulation of CIP2A by CagA, we used the MAP kinase inhibitor PD98059 to block MEK1 kinase activity, thereby inhibiting ERK1/2 phosphorylation (Alessi et al., 1995), SB203580 to block p38 kinase activity (Young et al., 1997), BAY11-7082 to inhibit the NF- $\kappa$ B pathway (Pierce et al., 1997) and PP1 to block Src-family tyrosine kinase activity (Hanke et al., 1996). Each signal inhibitor was incubated with gastric cells for $30 \mathrm{~min}$ prior to plasmid transfection. After $36 \mathrm{~h}$ transfection, while CIP2A upregulation by CagA was attenuated by PP1 (Src kinase) and PD98059 (MEK1), CIP2A upregulation was maintained using SB203580 (P38) and BAY11-7082 (NF- $\kappa$ B) (Fig. 3). In total, the results indicated that Src kinase and MEK/ERK pathways participated in CIP2A upregulation by CagA. In AGS cells, CagA protein was tyrosine phosphorylated by Src kinase, thereafter promoting the activated MEK/ERK pathway to upregulate CIP2A expression (Backert \& Selbach, 2008).

\section{Effect of CIP2A depletion and $\boldsymbol{H}$. pylori infection on Myc expression in AGS cells}

It has been reported that CIP2A depletion causes the downregulation of Myc expression in several cell lines ( $\mathrm{Li}$ et al., 2008). To investigate whether $H$. pylori infection could still upregulate Myc expression in AGS cells depleted
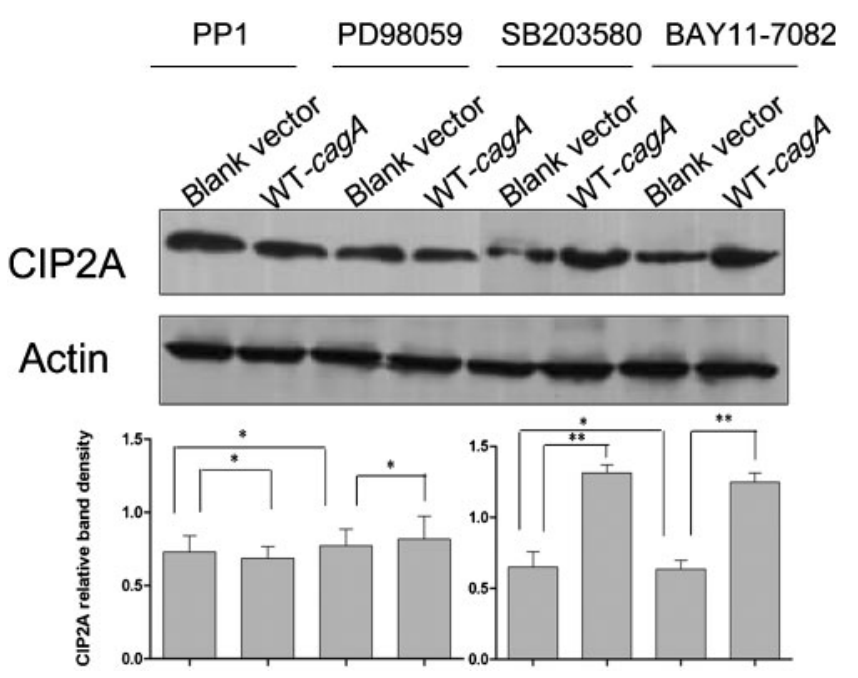

Fig. 3. Effects of signal inhibitors on CagA-induced CIP2A upregulation in AGS cells. AGS cells were transfected with blank vector or WT-cagA plasmid. The AGS cells were incubated with Src-family tyrosine kinase inhibitor PP1 $(20 \mu \mathrm{M})$, MEK1 inhibitor PD98059 $(50 \mu \mathrm{M})$, p38 MAP kinase inhibitor SB203580 (10 $\mu \mathrm{M})$ or NF- $\kappa$ B pathway inhibitor BAY11-7082 $(5 \mu \mathrm{M})$. Signal inhibitors were used $30 \mathrm{~min}$ before transfection and total cellular protein was extracted for Western blot analysis for the expression of CIP2A after transfection for 36 h. ${ }^{\star} P>0.05,{ }^{* *} P<0.01$. 
of CIP2A, we performed siRNA-mediated CIP2A depletion and $H$. pylori strain 26695 infection after depletion in AGS cells. We found that the Myc expression level was higher in cells treated with CIP2A depletion and $H$. pylori infection than in cells only treated with CIP2A depletion, and this level was lower in cells treated with CIP2A depletion and $H$. pylori infection than in those only treated with $H$. pylori infection (Fig. 4). The results suggest that $H$. pylori infection-induced Myc stabilization was partially attenuated by CIP2A depletion.

CIP2A has recently been observed to be overexpressed in head and neck squamous cell carcinoma, colon cancer (Junttila et al., 2007) and gastric cancer (Li et al., 2008). CIP2A overexpression stabilized the Myc protein (Khanna et al., 2009). The depletion of CIP2A inhibited growth and clonogenic capabilities, and induced senescence of AGS cells (Li et al., 2008). CIP2A is also known to act as an endogenous PP2A inhibitor (Junttila et al., 2007). H. pylori, a human gastric carcinogenic pathogen, has been classified as a class I carcinogenic factor by the World Health Organization and its long-term colonization in stomach initiates an increased risk of gastric cancer. Recent in vitro or in vivo data have demonstrated that $H$. pylori infection can upregulate cyclin D1 expression, stabilize Myc protein, increase $\beta$-catenin nuclear accumulation and induce release of cytokines such as interleukin-8 and CCL20 (Chang et al., 2006; Yang et al., 2003; Zhu et al., 2008; Franco et al., 2005; Kim et al., 2006; Tomimori et al., 2007). The expression level of murine double minute 2 was higher in gastric cancer than in chronic gastritis in $H$. pylori-infected gastric mucosa (Nakajima et al., 2009). However, up to now, the relationship between gastric carcinogenic pathogen $H$. pylori infection and gastric cancer oncoprotein CIP2A expression has never been studied. Thus we have explored the relationship between $H$. pylori infection, CIP2A expression and signalling pathways leading to gastric cancer.

Some former studies showed that $H$. pylori infection could upregulate cyclooxygenase 2 expression in vitro (Seo et al., 2007; Li et al., 2009). In our study, we verified that CIP2A expression in AGS cells was stimulated by $H$. pylori infection, and that this upregulation effect was not dependent on infection time but dependent on the cagA gene status of bacterial strains (Fig. 1). Kim et al. (2006) found that $H$. pylori CagA transfection of gastric epithelial cells induced IL-8 production, and Franco et al. (2005) found that CagA transfection increased $\beta$-catenin nuclear accumulation. In our study, we demonstrated that the CIP2A expression level was elevated by CagA transfection and that this elevation only occurred after tyrosine phosphorylation of CagA (Fig. 2). Moreover, Khanna et al. (2009) reported that Myc directly promoted CIP2A gene expression. Surprisingly, we found that CagA transfection also increased the Myc expression level (Fig. 2). These results offered more credible evidence for upregulation of CIP2A expression by CagA transfection. After translocation into epithelial cells, $H$. pylori CagA protein could activate four major pathways, including the MEK/ERK pathway, Src kinase, the NF- $\kappa$ B pathway and the p38 MAP kinase pathway (Backert \& Selbach, 2008). It was previously shown that signal inhibitors could be used to elucidate which pathway was involved in CagA-induced IL-8 production (Kim et al., 2006) or H. pylori infectioninduced cyclooxygenase 2 upregulation (Li et al., 2009). By using four corresponding signal inhibitors, we identified that Src kinase and MEK/ERK pathways were involved in CIP2A upregulation by CagA (Fig. 3). Taken together, CagA protein was tyrosine-phosphorylated by Src kinase, and thereafter activated the MEK/ERK pathway to upregulate CIP2A expression in AGS cells. We also found that $H$. pylori infection-induced Myc stabilization was partially dependent on CIP2A expression, and CIP2A depletion caused increased Myc degradation (Fig. 4).

The combination of data from our research and others led us to propose the signalling mechanism outlined in Fig. 5. Kim et al. (2006) reported that CagA transfection activated Src kinase, and Zhu et al. (2007) proved that CagA transfection and phosphorylated CagA protein (P-CagA) activated the ERK1/2 pathway. Based on these two findings, we used two corresponding signal inhibitors to block Src kinase or MEK1 kinase, and figured out the following mechanisms. After translocating into gastric epithelial cells through the TFSS, bacterial oncoprotein CagA was tyrosine-phosphorylated by Src kinase. After (a)

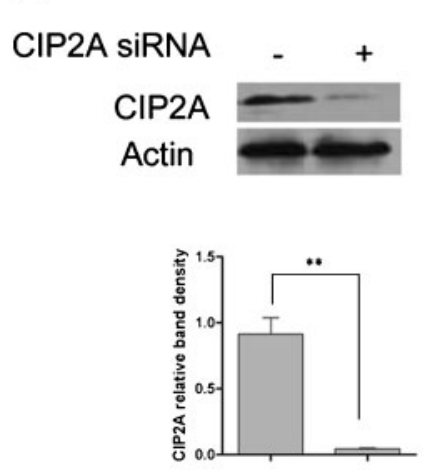

(b)
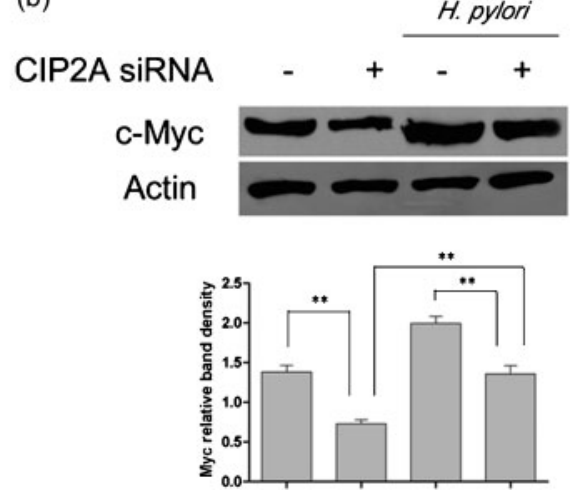

Fig. 4. Effects of CIP2A depletion and $H$. pylori infection on Myc expression in AGS cells. (a) AGS cells were transfected with control siRNA or CIP2A siRNA for $72 \mathrm{~h}$. Western blotting was used to verify the efficiency of CIP2A depletion. (b) After transfection with control siRNA or CIP2A siRNA for $72 \mathrm{~h}$, AGS cells were infected with $H$. pylori strain 26695 for 6 h. Western blotting was used for Myc expression analysis. ${ }^{\star \star} P<0.01$. 


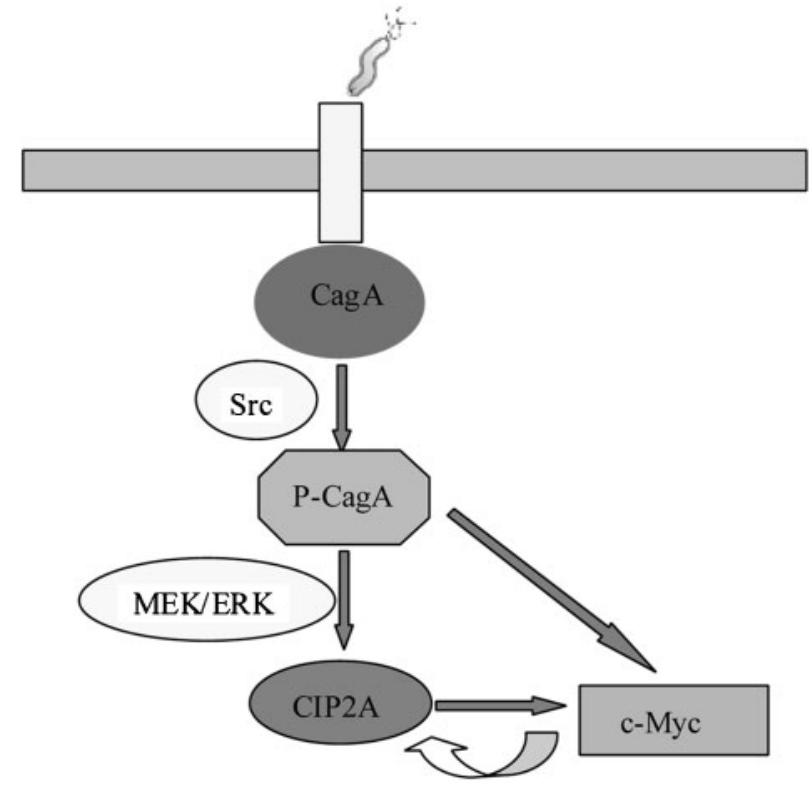

Fig. 5. Schematic model of the upregulation effect of $H$. pylori CagA on CIP2A. Bacterial oncoprotein CagA was translocated into gastric epithelial cells through the TFSS, and then activated Src kinase and was phosphorylated by Src kinase. After that, PCagA activated the MEK/ERK pathway and upregulated gastric oncoprotein CIP2A expression.

that, P-CagA activated the MEK/ERK pathway and upregulated gastric oncoprotein CIP2A expression. Upregulation of CIP2A expression by $H$. pylori provided a further explanation for the gastric carcinogenic effects of H. pylori infection.

Overall, this is the first time that the mechanism by which H. pylori infection and bacterial oncoprotein CagA upregulated CIP2A expression in gastric cell lines has been elucidated. CagA protein activated the Src and MEK/ERK signal pathways, resulting in the elevation of expression of CIP2A protein in AGS cells. This work has contributed to understanding the mechanism by which gastric tumours are caused by $H$. pylori infection in humans.

\section{ACKNOWLEDGEMENTS}

The study was supported by grants from the National Natural Science Foundation of China (Nos 30770118, 30972775, 30971151, 30800406 and 30800037), the National Basic Research Program of China (973 Program 2007CB914801) and the Science Foundation of Shandong Province (Nos ZR2009CZ001 and ZR2009CM002).

\section{REFERENCES}

Akopyants, N. S., Clifton, S. W., Kersulyte, D., Crabtree, J. E., Youree, B. E., Reece, C. A., Bukanov, N. O., Drazek, E. S., Roe, B. A. \& Berg, D. E. (1998). Analyses of the cag pathogenicity island of Helicobacter pylori. Mol Microbiol 28, 37-53.
Alessi, D. R., Cuenda, A., Cohen, P., Dudley, D. T. \& Saltiel, A. R. (1995). PD 098059 is a specific inhibitor of the activation of mitogenactivated protein kinase kinase in vitro and in vivo. J Biol Chem 270, 27489-27494.

Azuma, T., Yamazaki, S., Yamakawa, A., Ohtani, M., Muramatsu, A., Suto, H., Ito, Y., Dojo, M., Yamazaki, Y. \& other authors (2004). Association between diversity in the Src homology 2 domaincontaining tyrosine phosphatase binding site of Helicobacter pylori CagA protein and gastric atrophy and cancer. J Infect Dis 189, 820 827.

Backert, S. \& Meyer, T. F. (2006). Type IV secretion systems and their effectors in bacterial pathogenesis. Curr Opin Microbiol 9, 207-217.

Backert, S. \& Selbach, M. (2008). Role of type IV secretion in Helicobacter pylori pathogenesis. Cell Microbiol 10, 1573-1581.

Chang, Y. J., Wu, M. S., Lin, J. T., Pestell, R. G., Blaser, M. J. \& Chen, C. C. (2006). Mechanisms for H. pylori CagA-induced cyclin D1 expression that affect cell cycle. Cell Microbiol 8, 1740-1752.

Franco, A. T., Israel, D. A., Washington, M. K., Krishna, U., Fox, J. G., Rogers, A. B., Neish, A. S., Collier-Hyams, L., Perez-Perez, G. I. \& other authors (2005). Activation of $\beta$-catenin by carcinogenic Helicobacter pylori. Proc Natl Acad Sci U S A 102, 10646-10651.

Hanahan, D. \& Weinberg, R. A. (2000). The hallmarks of cancer. Cell $100,57-70$.

Handa, O., Naito, Y. \& Yoshikawa, T. (2007). CagA protein of Helicobacter pylori: a hijacker of gastric epithelial cell signaling. Biochem Pharmacol 73, 1697-1702.

Hanke, J. H., Gardner, J. P., Dow, R. L., Changelian, P. S., Brissette, W. H., Weringer, E. J., Pollok, B. A. \& Connelly, P. A. (1996). Discovery of a novel, potent, and Src family-selective tyrosine kinase inhibitor. Study of Lck- and FynT-dependent T cell activation. J Biol Chem 271, 695-701.

Hatakeyama, M. (2003). Helicobacter pylori CagA - a potential bacterial oncoprotein that functionally mimics the mammalian Gab family of adaptor proteins. Microbes Infect 5, 143-150.

Hatakeyama, M. (2004). Oncogenic mechanisms of the Helicobacter pylori CagA protein. Nat Rev Cancer 4, 688-694.

Houghton, J. \& Wang, T. C. (2005). Helicobacter pylori and gastric cancer: a new paradigm for inflammation-associated epithelial cancers. Gastroenterology 128, 1567-1578.

Junttila, M. R., Puustinen, P., Niemelä, M., Ahola, R., Arnold, H., Böttzauw, T., Alaaho, R., Nielsen, C., Ivaska, J. \& other authors (2007). CIP2A inhibits PP2A in human malignancies. Cell 130, 51-62.

Khanna, A., Böckelman, C., Hemmes, A., Junttila, M. R., Wiksten, J. P., Lundin, M., Junnila, S., Murphy, D. J., Evan, G. I. \& other authors (2009). MYC-dependent regulation and prognostic role of CIP2A in gastric cancer. J Natl Cancer Inst 101, 793-805.

Kim, S. Y., Lee, Y. C., Kim, H. K. \& Blaser, M. J. (2006). Helicobacter pylori CagA transfection of gastric epithelial cells induces interleukin-8. Cell Microbiol 8, 97-106.

Kwok, T., Zabler, D., Urman, S., Rohde, M., Hartig, R., Wessler, S., Misselwitz, R., Berger, J., Sewald, N. \& other authors (2007). Helicobacter exploits integrin for type IV secretion and kinase activation. Nature 449, 862-866.

Li, W., Ge, Z., Liu, C., Liu, Z., Björkholm, M., Jia, J. \& Xu, D. (2008). CIP2A is overexpressed in gastric cancer and its depletion leads to impaired clonogenicity, senescence, or differentiation of tumor cells. Clin Cancer Res 14, 3722-3728.

Li, Q., Liu, N., Shen, B., Zhou, L., Wang, Y., Wang, Y., Sun, J., Fan, Z. \& Liu, R. H. (2009). Helicobacter pylori enhances cyclooxygenase 2 expression via p38MAPK/ATF-2 signaling pathway in MKN45 cells. Cancer Lett 278, 97-103. 
Nakajima, N., Ito, Y., Yokoyama, K., Uno, A., Kinukawa, N., Nemoto, N. \& Moriyama, M. (2009). The expression of murine double minute 2 (MDM2) on Helicobacter pylori-infected intestinal metaplasia and gastric cancer. J Clin Biochem Nutr 44, 196-202.

Nguyen, L. T., Uchida, T., Murakami, K., Fujioka, T. \& Moriyama, M. (2008). Helicobacter pylori virulence and the diversity of gastric cancer in Asia. J Med Microbiol 57, 1445-1453.

Odenbreit, S., Puls, J., Sedlmaier, B., Gerland, E., Fischer, W. \& Haas, R. (2000). Translocation of Helicobacter pylori CagA into gastric epithelial cells by type IV secretion. Science 287, 1497-1500.

Peek, R. M. J. \& Blaser, M. J. (2002). Helicobacter pylori and gastrointestinal tract adenocarcinomas. Nat Rev Cancer 2, 28-37.

Pierce, J. W., Schoenleber, R., Jesmok, G., Best, J., Moore, S. A., Collins, T. \& Gerritsen, M. E. (1997). Novel inhibitors of cytokineinduced $\mathrm{I} \kappa \mathrm{B} \alpha$ phosphorylation and endothelial cell adhesion molecule expression show anti-inflammatory effects in vivo. J Biol Chem 272, 21096-21103.

Seo, J. H., Kim, K. H. \& Kim, H. (2007). Role of proteinase-activated receptor-2 on cyclooxygenase-2 expression in $H$. pylori-infected gastric epithelial cells. Ann N Y Acad Sci 1096, 29-36.

Stein, M., Rappuoli, R. \& Covacci, A. (2000). Tyrosine phosphorylation of the Helicobacter pylori CagA antigen after cag-driven host cell translocation. Proc Natl Acad Sci U S A 97, 1263-1268.

Stein, M., Bagnoli, F., Halenbeck, R., Rappuoli, R., Fantl, W. J. \& Covacci, A. (2002). c-Src/Lyn kinases activate Helicobacter pylori CagA through tyrosine phosphorylation of the EPIYA motifs. Mol Microbiol 43, 971-980.

Tomimori, K., Uema, E., Teruya, H., Ishikawa, C., Okudaira, T., Senba, M., Yamamoto, K., Matsuyama, T., Kinjo, F. \& other authors (2007). Helicobacter pylori induces CCL20 expression. Infect Immun $75,5223-5232$.
Yang, Y., Deng, C. S., Peng, J. Z., Wong, B. C., Lam, S. K. \& Xia, H. H. (2003). Effect of Helicobacter pylori on apoptosis and apoptosis related genes in gastric cancer cells. Mol Pathol 56, 19-24.

Yeh, E., Cunningham, M., Arnold, H., Chasse, D., Monteith, T., Ivaldi, G., Hahn, W. C., Stukenberg, P. T., Shenolikar, S. \& other authors (2004). A signalling pathway controlling c-Myc degradation that impacts oncogenic transformation of human cells. Nat Cell Biol 6, 308-318.

Yokoyama, K., Higashi, H., Ishikawa, S., Fujii, Y., Kondo, S., Kato, H., Azuma, T., Wada, A., Hirayama, T. \& other authors (2005). Functional antagonism between Helicobacter pylori CagA and vacuolating toxin VacA in control of the NFAT signaling pathway in gastric epithelial cells. Proc Natl Acad Sci U S A 102, 9661-9666.

Young, P. R., McLaughlin, M. M., Kumar, S., Kassis, S., Doyle, M. L., McNulty, D., Gallagher, T. F., Fisher, S., McDonnell, P. C. \& other authors (1997). Pyridinyl imidazole inhibitors of p38 mitogenactivated protein kinase bind in the ATP site. J Biol Chem 272, 1211612121.

Zhu, Y. L., Zheng, S., Qian, K. D. \& Fang, P. C. (2004). Biological activity of the virulence factor cagA of Helicobacter pylori Chin Med J (Engl) 117, 1330-1333.

Zhu, Y. L., Zheng, S., Du, Q., Qian, K. D. \& Fang, P. C. (2005). Characterization of CagA variable region of Helicobacter pylori isolates from Chinese patients. World J Gastroenterol 11, 880-884.

Zhu, Y., Wang, C., Huang, J., Ge, Z., Dong, Q., Zhong, X., Su, Y. \& Zheng, S. (2007). The Helicobacter pylori virulence factor CagA promotes Erk1/2-mediated Bad phosphorylation in lymphocytes: a mechanism of CagA-inhibited lymphocyte apoptosis. Cell Microbiol 9, 952-961.

Zhu, Y., Shu, X., Chen, J., Xie, Y., Xu, P., Huang, D. Q. \& Lu, N. H. (2008). Effect of Helicobacter pylori eradication on oncogenes and cell proliferation. Eur J Clin Invest 38, 628-633. 